\title{
Homogeneity Cues for Texel Size Estimation of Periodic and Near-Periodic Textures
}

\author{
Rocio A. Lizarraga-Morales, Raul E. Sanchez-Yanez, and Victor Ayala-Ramirez \\ Universidad de Guanajuato DICIS \\ Salamanca, Guanajuato, Mexico \\ rocio_lizarraga@laviria.org, \{sanchezy, ayalav\}@ugto.mx
}

\begin{abstract}
Texel size determination on periodic and near-periodic textures, is a problem that has been addressed for years, and currently it remains as an important issue in structural texture analysis. This paper proposes an approach to determine the texel size based on the computation and analysis of the texture homogeneity properties. We analyze the homogeneity feature computed from difference histograms, while varying the displacement vector for a preferred orientation. As we vary this vector, we expect a maximum value in the homogeneity data if its magnitude matches the texel size in a given orientation. We show that this approach can be used for both periodic and near-periodic textures, it is robust to noise and blur perturbations, and its advantages over other approaches in computation time and memory storage.
\end{abstract}

Keywords: Texel size detection, Textural periodicity, Difference histogram, Similarity test.

\section{Introduction}

Visual texture is a perceived property on the surface of all objects around us and can be an important reference for their characterization. From the structural point of view, it is widely accepted to define the texture as a conjunction of two components: i) a texture element (texel), which is the fundamental microstructure in the image [22, and ii) a set of rules for texel placement into the field of view. Such components can be used in several applications like shape from texture [2], texture synthesis [13114], texture compression [14], among others. Furthermore, the texel can be used as a reference to improve the performance in classification [612 and segmentation [18] tasks, and to achieve scale invariant texture analysis [21].

Texel size determination on periodic and near-periodic textures, is a problem that has been addressed for years. A typical approach is the use of the cooccurrence matrix (CM) proposed by Haralick 5]. This methodology has been widely used, mainly by exploiting its parametrization. Selkainaho et al. [17. detect texture periodicity by using $\kappa$ statistics emphasizing its computational advantages over $\chi^{2}$ statistics. Oh et al. [16], have proposed a fast determination of textural periodicity using a binary co-occurrence matrix, improving the 
processing time in comparison with the CM in gray level images. But Recently, other non-CM-based approaches have been proposed. Grigorescu and Petkov [3], determine texel size of periodic and near-periodic images based on the calculation of Renyi's generalized entropies by assuming a square texel for all textures. Other studies for texture periodicity detection are found in the literature, we can mention those based on the wavelet transform [8, autocorrelation [109], or regular bands [15].

In this work, we explore the use of difference histograms, originally proposed by Unser [19], to detect the texel size of both periodic and near-periodic visual textures. On the proposed approach, we specifically use the homogeneity property computed from the difference histogram (DH). Our method exploits the fact that the homogeneity attains its maximum value when the parameter of $\mathrm{DH}$ takes the value of the texel size or any positive integer multiple of it. Moreover, DH computation can be done more efficiently than CM both in memory usage and algorithmic complexity.

This paper is structured as follows: Section 2 describes the homogeneity feature used for the proposed method and our approach to estimate the texel size. In Section 3 we present the experiments and results performed to validate our method on a set of corrupted textures and natural near-periodic textures. In this section, it is also presented a computation time comparison with other approaches. Section 4 presents a summary of this work and our conclusion.

\section{Texel Size Estimation Using Homogeneity Cues}

Sum and Difference Histograms (SDH), were introduced by Unser [19] as an alternative to the usual co-ocurrence matrix (CM). Unlike the CM that occupies $K^{2}$ memory elements for an image with $K$ gray levels, SDH present memory storage reduction, since they only occupy two arrays of $2 K-1$ integers. To obtain the $\mathrm{SDH}$, let us define an image $I$ of $M \times N$ pixels size, which has $K$ gray levels $k=0,1, \ldots, K-1$. Consider a pixel positioned in the coordinates $(m, n)$ with intensity $I_{m, n}$ and a second pixel in the relative position $\left(m+v_{m}, n+v_{n}\right)$ with intensity $I_{m+v_{m}, n+v_{n}}$. The non-normalized sum and differences, of two pixels associated with the relative displacement vector $V=\left(v_{m}, v_{n}\right)$, are defined as:

$$
\begin{aligned}
& s_{m, n}=I_{m, n}+I_{m+v_{m}, n+v_{n}} \\
& d_{m, n}=I_{m, n}-I_{m+v_{m}, n+v_{n}} .
\end{aligned}
$$

Sum and Difference Histograms $h_{s}$ and $h_{d}$, with displacement vector $V=\left(v_{m}, v_{n}\right)$ over the image domain $D$, are defined as:

$$
\begin{aligned}
h_{s}(i) & =\operatorname{Card}\left\{(m, n) \in D, s_{m, n}=i\right\} \\
h_{d}(j) & =\operatorname{Card}\left\{(m, n) \in D, d_{m, n}=j\right\} .
\end{aligned}
$$

The normalized sum and differences histograms are estimations of the sum and difference probability functions defined by $P_{s}(i)$ and $P_{d}(j)$. 
A number of features computed from the probability functions have been proposed to be used as textural features, but the result largely depends on the size of the displacement vector $V$. These features for texture description have been proposed as a simplification of those proposed by Haralick [5]. In this study we specifically use the homogeneity, defined as:

$$
\text { homogeneity }=G=\sum_{j} \frac{1}{1+j^{2}} \cdot P_{d}(j)
$$

This feature has two advantages, it is normalized in the range [0, 1], and it only uses the difference probability distribution, reducing memory and time consumption.

In this paper, we analyze the difference histogram behavior to estimate the period of a given texture. Differences $d_{m, n}$, resulting from the use of a $V$ that matches the period only take values of zero, as the reference pixel $I(m, n)$ and the relative pixel $I\left(m+v_{m}, n+v_{n}\right)$ have the same value. If the difference histogram has recorded only one value, the homogeneity function $G$ reaches its maximum value.

Estimation of the period in a given direction can be done by setting a component to 0 . That is, to detect the horizontal period of the texture, we set $v_{n}=0$. We can detect periodicity values for $T_{m}$ ranging from 2 to $\frac{M}{2}$. In a similar way, the detection of the texture period in the vertical direction $T_{n}$ can be done by setting $v_{m}=0$. We can detect values of $T_{n} \in\left[2, \frac{N}{2}\right]$.

As an example of this method, we show an artificial and periodic texture with a texel size of $60 \times 40$ pixels in Fig. 1a, and its homogeneity plot in Fig. 10. We can see the periodicity of both plots as they present maxima values at the corresponding texel size multiples, 60 and 120 for horizontal detection, and 40, 80 and 120 for vertical detection.

\section{Experiments and Results}

In this section, we present an experimental evaluation of the proposed approach. We have separated our experiments in two parts: the first part consists in evaluating our approach with a corrupted regular pattern and the second part consists in using natural near-periodic images as inputs to our method.

\subsection{Texel Size Estimation of a Corrupted Regular Pattern}

In order to evaluate the limits of our approach under different conditions, we have corrupted a periodic texture pattern (Fig. 17) with varying blur and noise levels. With this pattern, we have a benchmark, as we know that the pattern has a texel size of $60 \times 40$ pixels.

Blur. In this section, we have applied a simple low-pass filter a number of times in order to obtain a blur effect. The blur effect causes the loss of detail in the 


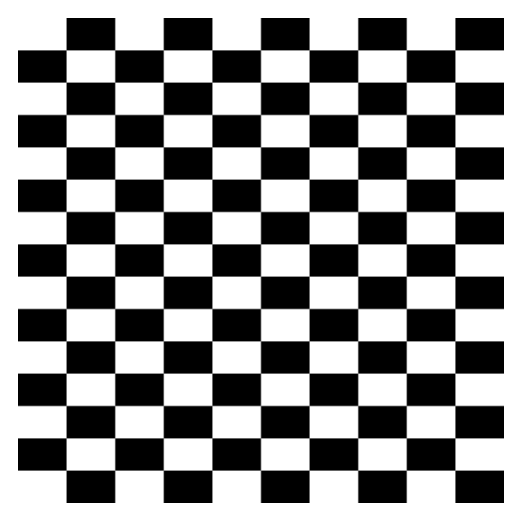

(a)

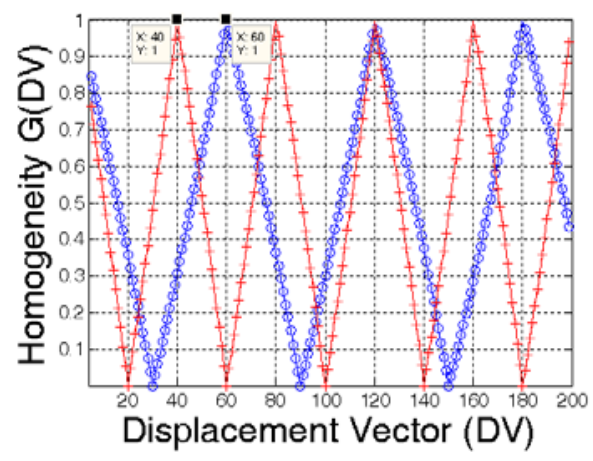

(b)

Fig. 1. An artificial texture (a) and its homogeneity function (b) in both, horizontal (o) and vertical $(+)$ directions

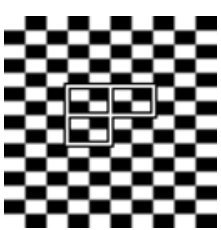

(a)

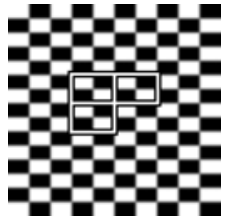

(b)

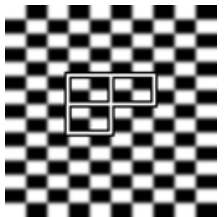

(c)

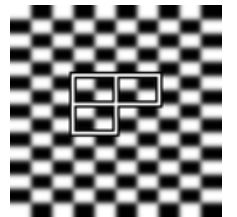

(d)

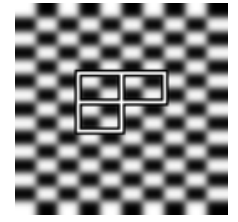

(e)

Fig. 2. Synthetic images with blur variations and the estimated texel size $(60 \times 40$ in all cases). The blur filter is applied (a) 2 times, (b) 4 times, (c) 8 times, (d) 16 times, (e) 32 times.

image, making difficult the texel detection. This filter has been applied 2, 4, 8, 16 and 32 times. Resulting images are shown in Fig. 2, In this figure, the detected texel is highlighted twice in each direction for comparison purposes. As we can see, the texel is accurately detected despite the blur effect in all the images, so we can infer that these blur levels do not affect the performance and accuracy of our approach.

Salt and Pepper Noise. In order to evaluate the performance of our approach with noise, we have corrupted the same periodic texture pattern with salt and pepper noise in different occupancy levels. Image noise is usually regarded as undesirable, which is why it is important to evaluate our approach under noisy conditions. Occupancy levels of noise considered in tests are 5\%, 10\%, 20\%, 40\% and $80 \%$, but in order to extend the test we have randomly built 100 images for each occupancy level. These 500 images are used as inputs to our method. A sample of the aspect of the resulting images is shown in Fig. 3, where the texel detected is also shown for each image. Results in terms of percentage of correctly detected texels show that our approach detects properly the texel size 


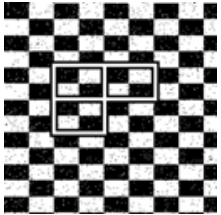

(a)

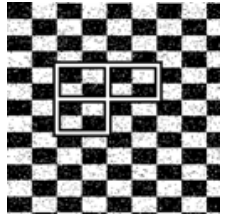

(b)

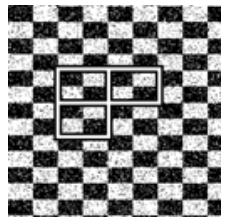

(c)

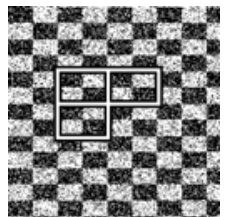

(d)

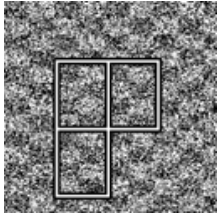

(e)

Fig. 3. A sample of the image corrupted with each noise occupancy level and the texel size detected by our method. Noise occupancy levels are (a) 5\%, (b) 10\%, (c) 20\%, (d) $40 \%$, (e) $80 \%$.

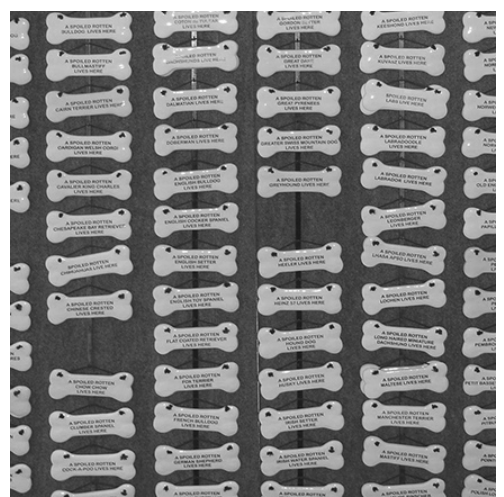

(a)

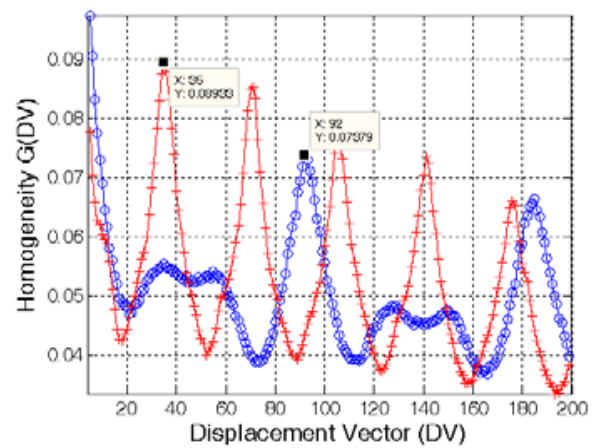

(b)

Fig. 4. A natural texture (a) and its homogeneity function (b) in both directions horizontal (o) and vertical (+)

with $5 \%$ and $10 \%$ noise occupancy in the 100 images. With occupancy levels of $20 \%$ and $40 \%$ an error occurs in only 6 images. With $80 \%$ of noise occupancy, in 62 times the texels were accurately detected. Most of the errors are related with the detection of multiples of the texel size, as we can see in Fig. 3e, where our system detects a texel size of $60 \times 80$.

\subsection{Texel Size Estimation of Natural Images}

After tests on synthetic images, we have evaluated the performance of our approach using near-periodic natural images. Near-periodic textures are those that are not strictly periodic, showing irregularities in color, intensity, noise, global or local deformations, resolution, etc. 20] presenting a challenge for any system. Texel size of a periodic texture pattern is estimated by finding the first maximum, but it is more difficult when a natural texture is analyzed (see Fig. 4). In order to generalize our method, we say that texel size is estimated by the $V$ where the global maximum from homogeneity functions is found. 


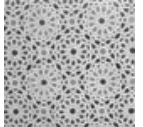

(a)

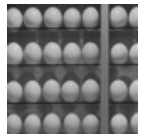

(i)

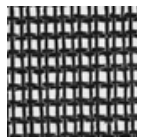

(q)

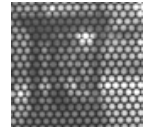

(b)

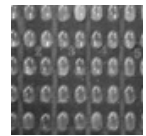

(j)

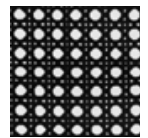

(r)

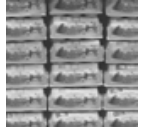

(c)

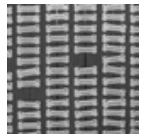

(k)

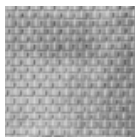

(s)

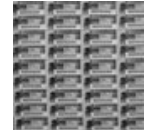

(d)

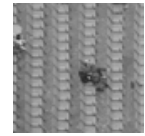

(l)

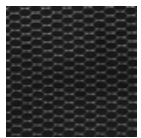

$(\mathrm{t})$

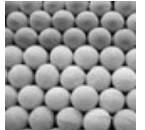

(e)

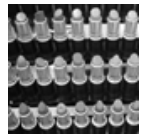

(m)

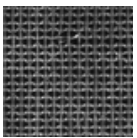

(u)

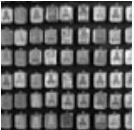

(f)

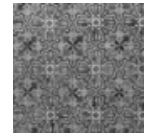

(n)

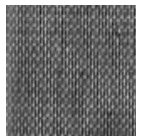

( v)

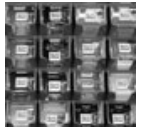

(g)

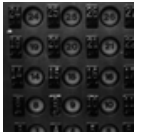

(o)

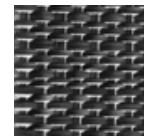

(w)

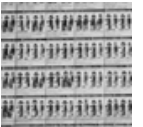

(h)

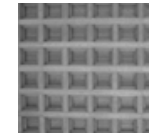

(p)

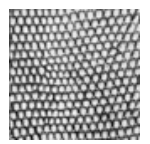

(x)

Fig. 5. Natural images set used in experiments

We have evaluated the performance of our method using a set of 16 natural images extracted from the album proposed by Klette [7] and 8 images from the Brodatz album 11. At a first glance, these 24 textures (see Fig. 5) seem to be periodic. Nevertheless, a thorough inspection shows that the texture periodicity descriptors (texel size, texel shape and texel placement rules) and the intensity properties (illumination, blur, contrast, noise), vary through the image because of its natural origin.

Table 1 presents the results of texel size estimation using our method for each image. In order to evaluate the goodness of our method, we have implemented a simple texture synthesis by using a tiling algorithm. In this algorithm, a sample is tiled up to fill an image of the same dimensions of the original texture. Some qualitative results are shown in Fig. 6, where the original image is given, highlighting the detected texel. These results show that even when a tiling algorithm seems not appropriate for near-periodic textures, the accuracy in our texel detection allows to both textures, original and synthetic, seem not identical but quite similar.

A quantitative evaluation is carried out with a cosine similarity measure in order to quantify the similarity between the original image and the synthetic image. Cosine similarity measure is a common vector similarity metric, that guarantees, as do other similarity metrics, that $0 \leq \cos \left(h_{1}, h_{2}\right) \leq 1$, so we can have an intuitive result where 1 is the value for two images that match exactly. Cosine similarity metric is computed as: $\cos \left(h_{1}, h_{2}\right)=\frac{\sum_{k} h_{1}(k) h_{2}(k)}{\sqrt{\sum_{k} h_{1}^{2}(k) \sum_{k} h_{2}^{2}(k)}}$, where $h_{1}$ and $h_{2}$ are representative histograms of two images. In this paper, each image is represented by the Sum and Difference Histograms with different arbitrary displacement vectors. Therefore, we have two similarity values and the final result is the average of these two values. 


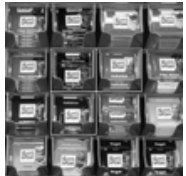

(a) $112 \times 107$

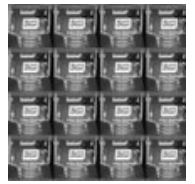

(b)

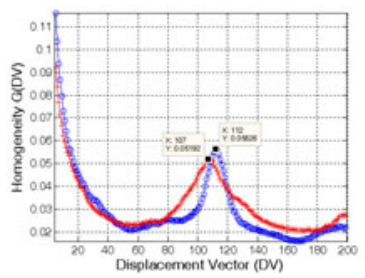

(c)

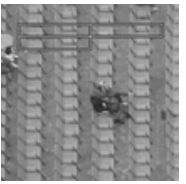

(a) $17 \times 41$

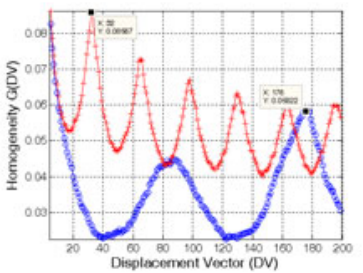

(c)

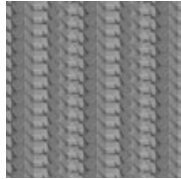

(b)

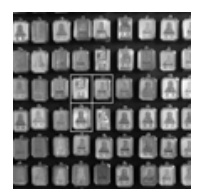

(a) $51 \times 71$

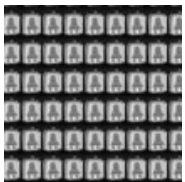

(b)

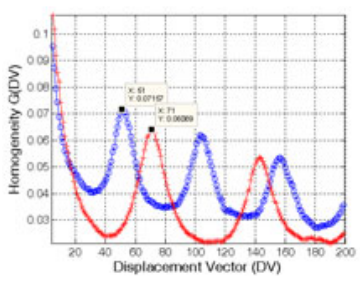

(c)

Fig. 6. Some qualitative results. (a) Original image with the estimated texel overlaid, (b) Synthetic texture resulting from the tiling of the detected texel, and (c) corresponding homogeneity plot for the image in (a).

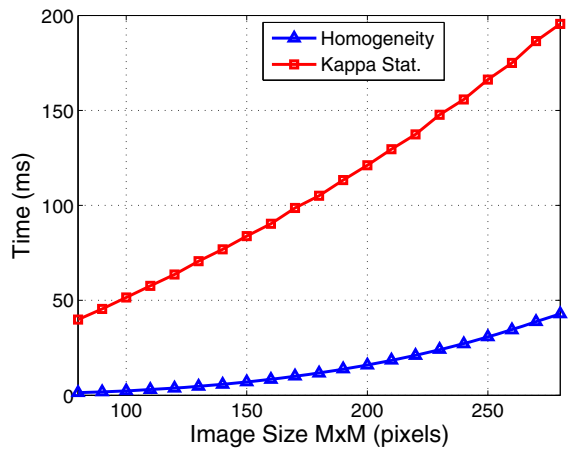

(a)

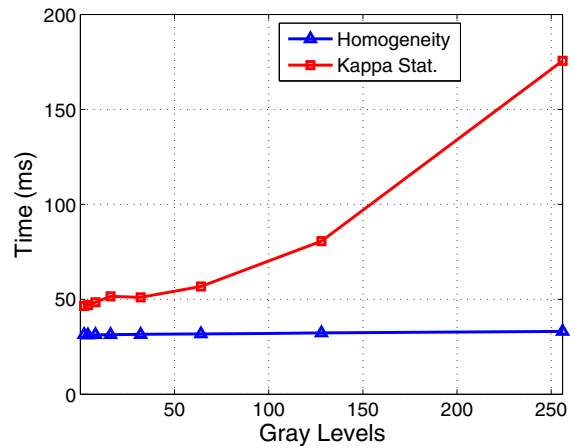

(b)

Fig. 7. Computation time of the proposed algorithm and the Kappa statistics for (a) Different Image Sizes and (b) Varying Gray Levels in the image

Results of cosine similarity measure are also presented in Table 1 . These values show that the synthetic image is very similar to the original one with an average value of 0.8879 ( $88.8 \%$ similar). Synthetic images corresponding with (a), (c), (d), (f), (j), (k), (l), (n), (q), (r) and (u), exhibit good similarity values, higher than 0.9 , this is, images are similar in more that $90 \%$. The lowest value of 0.704 (marked in Table 1 in bold) is obtained with the image (i). In general, there are slight differences between the original and synthetic images, due to natural irregularities of texture. 
Table 1. Texel size determination for textures in Fig. 5 and the similarity measure for the corresponding synthesized texture

\begin{tabular}{|ccc||ccc|}
\hline Texture & Texel Size & Similarity & Texture & Texel Size & Similarity \\
\hline \hline (a) & $194 \times 198$ & 0.967 & $(\mathrm{~m})$ & $56 \times 137$ & 0.846 \\
\hline (b) & $27 \times 47$ & 0.880 & $(\mathrm{n})$ & $151 \times 153$ & 0.913 \\
\hline (c) & $180 \times 78$ & 0.947 & $(\mathrm{o})$ & $123 \times 97$ & 0.842 \\
\hline (d) & $117 \times 47$ & 0.973 & $(\mathrm{p})$ & $81 \times 78$ & 0.846 \\
\hline (e) & $84 \times 132$ & 0.829 & $(\mathrm{q})$ & $33 \times 33$ & 0.915 \\
\hline (f) & $51 \times 71$ & 0.892 & (r) & $38 \times 38$ & $\mathbf{0 . 9 9 0}$ \\
\hline (g) & $112 \times 107$ & 0.920 & $(\mathrm{~s})$ & $25 \times 31$ & 0.872 \\
\hline (h) & $22 \times 95$ & 0.825 & $(\mathrm{t})$ & $41 \times 17$ & 0.877 \\
\hline (i) & $64 \times 117$ & $\mathbf{0 . 7 0 4}$ & $(\mathrm{u})$ & $32 \times 29$ & 0.973 \\
\hline$(\mathrm{j})$ & $110 \times 91$ & 0.951 & $(\mathrm{v})$ & $14 \times 19$ & 0.805 \\
\hline$(\mathrm{k})$ & $92 \times 35$ & 0.918 & $(\mathrm{w})$ & $69 \times 28$ & 0.875 \\
\hline$(\mathrm{l})$ & $176 \times 32$ & 0.980 & $(\mathrm{x})$ & $15 \times 31$ & 0.770 \\
\hline
\end{tabular}

\subsection{Computation Time Evaluation}

In this section, we have compared in computation time our algorithm with different well-known approaches. The tested approaches are: a CM-based method using $\kappa$ statistics, an auto-correlation method and the method based on Renyi's generalized entropies. We have evaluated these methods in their computation time dependance on (1) Image size and (2) Gray levels in an image. For each method in both tests, we obtain the average computation time of 100 executions.

For the first experiment (see Fig. 7 a) we have tested square images of varying sizes of $M \times M$ pixels, with $M \in[80,280]$, and in the second experiment (see Fig. $7 \mathrm{~b})$ we have tested images of $256 \times 256$ pixels size with varying the gray levels $K$ with $K \in\{2,4,8,16,32,64,128,256\}$. As can be seen in the figures, the time curves are noticeably different. Our approach time consumption is considerably lower than the CM-based method in both cases, depending on the image size and depending on the gray levels. We can observe that the corresponding curve for the CM-based method increases more and faster than the curve for our method. Results of auto-correlation-based and Renyi's generalized entropies-based methods were obtained but they have not been plotted because they are out of scale. These methods are time-consuming, they are measured in minutes for an image of $256 \times 256$ pixels size.

All of our data were obtained by using non-optimized $\mathrm{C}$ implementations on an ordinary Intel(R) Core(TM)2 Duo $3.05 \mathrm{GHz}$ CPU with $2 \mathrm{~GB}$ of RAM.

\section{Summary and Conclusions}

Texel size detection is a classical problem in structural texture analysis. In this paper, the use of homogeneity cues to detect the texel size in periodic, corrupted 
periodic and near-periodic textures has been discussed. Homogeneity was presented as a function of a displacement vector, which determines the histogram to be used. When the displacement vector matches the texel size, the homogeneity reaches its maximum value. With this in mind, we can easily detect the basic pattern repeated along a specific texture surface. Natural textures lose periodicity because of usual surface irregularities. However, homogeneity function still has local maxima corresponding to the texel size and multiples.

The algorithm, yet simple, is a robust method with respect to blur distortions and noise corruption near to $80 \%$. This robustness is also shown using texture synthesis with near-periodic natural textures as inputs. In most cases, we obtain a good similarity index between the original image and the synthesized one. Other advantage of the proposed method is the ability of detection of period in both horizontal and vertical directions. Therefore, we can easily detect both square and rectangular texels. This approach, is fast enough to be considered for practical applications, since it takes 0.015 s to detect the texel size in a $200 \times 200$ pixels size and 256 gray levels image with a non-optimized implementation.

\section{Acknowledgments}

R.A. Lizarraga-Morales acknowledges the Mexican CONACyT and CONCyTEG for the financial support via scholarships, grant numbers 206622 and 08-16-K119139 , respectively.

\section{References}

1. Brodatz, P.: Textures: A Photographic Album for Artists and Designers. Dover Publications, New York (1966)

2. Forsyth, D.: Shape from texture without boundaries. In: Heyden, A., Sparr, G., Nielsen, M., Johansen, P. (eds.) ECCV 2002. LNCS, vol. 2352, pp. 225-239. Springer, Heidelberg (2002)

3. Grigorescu, S., Petkov, N.: Texture analysis using Renyi's generalized entropies. In: Proc. of the IEEE (ICIP 2003), vol. 1, pp. 241-244. IEEE, Los Alamitos (2003)

4. Gui, Y., Ma, L.: Periodic pattern of texture analysis and synthesis based on texels distribution. The Visual Computer 26(6-8), 951-964 (2010)

5. Haralick, R.: Statistical and Structural Approaches to texture. In: Proc. on the IEEE 4th. Int. Joint Conf. Pattern Recognition, pp. 45-60 (1979)

6. Jan, S.R., Hsueh, Y.C.: Window-size determination for granulometrical structural texture classification. Pattern Recogn. Lett. 19(5-6), 439-446 (1998)

7. Klette, R.: Basic multimedia imaging (2002), http://www.cs.auckland.ac.nz/ rklette/TeachAuckland.html/ mm/Pictures/220Textures_RK.zip

8. Lee, K.L., Chen, L.H.: A new method for extracting primitives of regular textures based on wavelet transform. Int. J. of Patt. Recogn. and Artif. Intell. 16, 1-25 (2002)

9. Leu, J.G.: On indexing the periodicity of image textures. Image and Vision Computing 19(13), 987-1000 (2001) 
10. Lin, W.C., Hays, J., Wu, C., Liu, Y., Kwatra, V.: Quantitative Evaluation of Near Regular Texture Synthesis Algorithms. In: IEEE Computer Society Conf. on Computer Vision and Pattern Recognition, vol. 18(5), pp. 427-434 (2006)

11. Liu, Y., Lin, W.C., Hays, J.: Near-regular texture analysis and manipulation. In: SIGGRAPH 2004, pp. 368-376. ACM Press, New York (2004)

12. Lizarraga-Morales, R.A., Sanchez-Yanez, R.E., Ayala-Ramirez, V.: Optimal spatial predicate determination of a local binary pattern. In: Proc. of the (VIIP 2009), pp. 41-46. Acta Press (2009)

13. Lobay, A., Forsyth, D.: Recovering shape and irradiance maps from rich dense texton fields. In: Proc. of the (CVPR 2004), pp. 400-406 (2004)

14. Menegaz, G., Franceschetti, A., Mecocci, A.: Fully automatic perceptual modeling of near regular textures. In: SPIE Human Vision and Electronic Imaging XII, vol. 6492, pp.64921B.1-64921B.12. SPIE, San Jose (2007)

15. Ngan, H.Y.Y., Pang, G.K.: Regularity analysis for patterned texture inspection. IEEE Trans. on Automation Science and Engineering 6(1), 131-144 (2009)

16. Oh, G., Lee, S., Shin, S.Y.: Fast determination of textural periodicity using distance matching function. Pattern Recogn. Lett. 20(2), 191-197 (1999)

17. Selkainaho, K., Parkkinen, J., Oja, E.: Comparison of $\chi^{2}$ and $\kappa$ statistics in finding signal and picture periodicity. In: Proc. 9th Int. Conf. Patt. Recogn., pp. 1221-1224 (1988)

18. Todorovic, S., Ahuja, N.: Texel-Based texture Segmentation. In: Proc. of the (ICCV 2009), pp. 841-848 (2009)

19. Unser, M.: Sum and difference histograms for texture classification. IEEE Trans. on Pattern Anal. Mach. Intell. 8(1), 118-125 (1986)

20. Liu, Y., Tsin, Y., Lin, W.C.: The Promise and Perils of Near-Regular Texture. Int. J. of Computer Vision 62(1-2), 145-159 (2005)

21. Zhang, J., Tan, T.: Brief review of invariant texture analysis methods. Pattern Recognition 35, 735-747 (2002)

22. Zhu, S., Guo, C., Wang, Y., Xu, Z.: What are Textons? Int. J. of Computer Vision 62, 121-143 (2005) 